\title{
Vitrectomy may increase the sensitivity of the retinal pigment epithelium to external stimuli
}

\author{
Abdullah Kaya ${ }^{1} \cdot$ Yakup Aksoy $^{2}$ \\ Received: 18 February 2016 / Accepted: 15 March 2016/Published online: 28 March 2016 \\ (C) Springer-Verlag Berlin Heidelberg 2016
}

Dear Editor,

We read with great interest the article titled "The effectiveness of vitrectomy for diffuse diabetic macular edema may depend on its preoperative optical coherence tomography pattern" by Ichiyama et al. [1]. The authors evaluated the influence of optical coherence tomography (OCT) patterns of diffuse diabetic macular edema (DDME) on the effectiveness of vitrectomy. They classified DDME as sponge-like diffuse retinal thickening (SRDT), cystoid macular edema (CME) and serous retinal detachment (SRD), and found that the success rate was higher in patients with SRD. Serous retinal detachment may develop as a result of an impairment of the outer blood retinal barrier or in-pump function of retinal pigment epithelium (RPE). If there were an impairment in the outer blood retinal barrier, we would expect anti-VEGF drugs to be effective in curing SRD. However, anti-VEGF was found to be less effective in this type of DDME [2]. The primary reason for the subretinal fluid (SRF) accumulation, therefore, appears to be ineffective pump function of the RPE. The authors also discussed the impairment of RPE function as a possible cause

Abdullah Kaya

abdullahkayamd@gmail.com

1 Department of Ophthalmology, Anıttepe Military Dispensary, Ankara, Turkey

2 Department of Ophthalmology, Girne Military Hospital, Girne, Cyprus of SRD, suggesting that hypoxia may reduce pump function. However, the choroid has a high blood flow and seems to carry sufficient oxygen to the RPE. There may be another relationship between the removal of vitreous and improved RPE function. We suggest that the increased sensitivity of RPE to external impulses after vitrectomy may be a reason. RPE reacts to external stimuli. For example, subthreshold diode laser micropulse photocoagulation enhances RPE function. Because of its colloid and dense structure, the vitreous appears to absorb vibrations. After vitrectomy, sound waves or mechanical vibrations generated from eye motion or blinking may easily reach and stimulate the RPE.

\section{Compliance with ethical standards}

Founding None.

Conflicts of interest None.

\section{References}

1. Ichiyama Y, Sawada O, Mori T, Fujikawa M, Kawamura H, Ohji M (2016) The effectiveness of vitrectomy for diffuse diabetic macular edema may depend on its preoperative optical coherence tomography pattern. Graefes Arch Clin Exp Ophthalmol. doi:10.1007/ s00417-015-3251-4

2. Shimura M, Yasuda K, Nakazawa T, Hirano Y, Sakamoto T, Ogura Y, Shiono T (2011) Visual outcome after intravitreal triamcinolone acetonide depends on optical coherence tomographic patterns in patients with diffuse diabetic macular edema. Retina 31(4):748-754 